\title{
Theoretical and Experimental Analysis on the Thickness-Controlled Residual Stress during Drying of Solvent-Absorbed Polymer Films
}

\author{
Kwang Seung PARK, Young Don KwON, ${ }^{*}$ and Dukjoon $\mathrm{KIM}^{\dagger}$ \\ Department of Chemical Engineering and Polymer Technology Institute, Sungkyunkwan University, Suwon, Kyunggy 440—746, Korea \\ ${ }^{*}$ Department of Textile Engineering, Sungkyunkwan University, Suwon, Kyunggy 440—746, Korea
}

(Received August 18, 2000; Accepted March 30, 2001)

\begin{abstract}
Thickness-controlled residual stress was experimentally measured during drying of polyimide films using interferometer and bending beam systems. The local solvent concentration and temperature inside films were theoretically predicted using Fick's diffusion and Fourier's heat transfer equations. Introduction of bending moment theory made it possible to develop equations relating the residual stress with solvent concentration in films. The experimental stress behavior was in good agreement with the theoretical one, and the modulus of polyimide/NMP systems was determined from the best fit of two results.

KEY WORDS Diffusion / Residual Stress / Drying Process / Polymer Films / Modulus /
\end{abstract}

Thin polymer films have been applied for corrosion protective coating, microlithography, packaging of semi-conductors, membranes, controlled delivery systems, and so on. ${ }^{1,2}$ Polymer films are usually manufactured by a film casting method, where the solvent in polymer solution coated on a circuit substrate diffuses through the film and evaporates into atmosphere.

During drying of polymer films, solvent diffusion and evaporation lead to variation of stress as well as concentration, thickness, and physical properties of films. In industrial film manufacturing, the residual stress associated with the solvent remaining in film affects their final performance, sometimes leading to severe deterioration by cracking and delamination. For this reason, the experimental and theoretical analysis of residual stress is essential for the safe fabrication and application of polymer films.

Recently, we in-situ analyzed the imidization extent, thickness, and residual stress of polyimide (PI) films during curing using several techniques, and the effects of scanning rate and pre-baking time were studied. ${ }^{3}$ The stress was determined considering not only the typical radius of curvature, but also the film thickness. The stress was significantly controlled not only by the imidization kinetics, but also by the film thickness. Also, we measured the film thickness change during drying of $N$-methyl pyrollidone (NMP) - absorbed PI films, and analyzed the experimental data theoretically with the extraction of diffusion and mass transfer coefficients. ${ }^{4,5}$ As continuation of the previous work, we measured the residual stress during drying of PI/NMP films in this study, and developed equations that modeled experimental behavior in relation to diffusion. In addition, we tested the validity of the theory by its comparison with experimental data with the determination of Young's modulus of the film. PI/NMP systems are widely used in microelectronics fabrication and also have many other applications..$^{6-8}$

\section{EXPERIMENTAL}

\section{Film Preparation}

The concentration of poly(amic acid) (PAA) solution (Pyralin ${ }^{\circledR}$ PI2545, DuPont) employed as a precursor of PI was $13.8 \mathrm{wt} \%$, in which NMP was the solvent and the monomers were pyromellitic dianhydride (PMDA) and $4,4^{\prime}$-oxydianiline (ODA).

PAA was coated on the $360 \mu \mathrm{m}$-thickness silicon wafer $(70 \times 9 \mathrm{~mm})$ for $30 \mathrm{~s}$ at $1800 \mathrm{rpm}$ using a spin coater (model 1-EC101DT-R485, Headway, U. S. A.). The film-coated substrate was stored in the drying oven at $80^{\circ} \mathrm{C}$ for $60 \mathrm{~min}$ in order to remove excess amount of NMP. PI films were cured by increasing temperature from room temperature to $400^{\circ} \mathrm{C}$ under $\mathrm{N}_{2}$ atmosphere at the ramping rate of $4^{\circ} \mathrm{C}$ min. The cured PI film was immersed in NMP at $60^{\circ} \mathrm{C}$ until equilibrium amount of solvent was absorbed.

\section{Measurement of Film Thickness}

Laser interferometer was used in order to measure the film thickness $L_{\mathrm{f}}$ during drying of films. It was assumed that film thickness was the sum of pure polymer thickness $L_{\mathrm{p}}$ and solvent thickness $L_{\mathrm{s}}$, and the refractive index of solvent absorbed film was the volume average of the respective indices of components. Total film thickness change during drying was determined by

${ }^{\dagger}$ To whom correspondence should be addressed (Fax : 82-31-290-7272, E-mail : djkim@yurim.skku.ac.kr). 


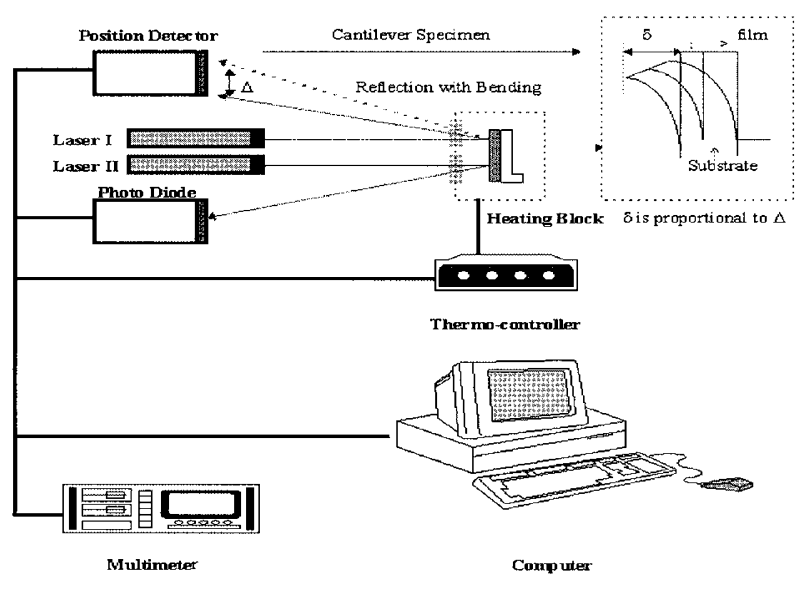

Figure 1. Schematic apparatus for in-situ stress and film thickness measurements using the bending-beam and interferometer systems.

counting the number of maximum interfered beam intensities, as each interval between the consecutive maximum peaks corresponds to the reduction of film thickness given by eq $1^{9}$ :

$$
\frac{\Delta L_{\mathrm{f}}}{\text { fringe }}=\left(\frac{\mathrm{d} \phi / \mathrm{d} L_{\mathrm{f}}}{2 \pi}\right)^{-1}=\frac{\lambda_{\text {beam }}}{2 n_{\mathrm{s}}}
$$

Here, $\lambda_{\text {beam }}$ is the wavelength of laser beam, $n_{\mathrm{S}}$ is the refractive index of solvent, and $\phi$ the phase difference between the lights reflected from film surface and from film/substrate interface.

Figure 1 schematically shows the interferometer system employed in the current study. Inside the heating chamber, the NMP-absorbed PI film was placed vertically. The film surface was irradiated with the laser beam with the wavelength of $632.8 \mathrm{~nm}$ generated by the $1 \mathrm{~mW}$ He-Ne Laser II (model 1507-0, Uniphase, U. S. A.). The light beams reflected from film surface and film/wafer interface were detected by a silicone photodiode (model s2386-8 K, Hamamatsu, U. S. A.). The data were digitized to be stored in a personal computer.

\section{Measurement of Residual Stress}

Since the elastic modulus of wafer substrate $E_{\text {sub }}$ is much higher than that of polymer film $E_{\mathrm{f}}$, the residual stress $\sigma_{\mathrm{f}}$ can be determined by the following equation ${ }^{10}$ involving the radius of curvature of bending beam $R$ and film thickness $L_{\mathrm{f}}$ :

$$
\sigma_{\mathrm{f}}=\frac{1}{6 R} \times \frac{E_{\mathrm{sub}} L_{\mathrm{sub}}^{2}}{\left(1-\gamma_{\mathrm{sub}}\right) L_{\mathrm{f}}}=\frac{E_{\mathrm{sub}}}{1-\gamma_{\mathrm{sub}}} \times \frac{L_{\text {sub }}^{2}}{3 R^{2} L_{\mathrm{f}}} \times \delta
$$

Here, $L_{\text {sub }}$ and $\gamma_{\text {sub }}$ indicate the thickness and Poisson's ratio of substrate, respectively. Also $R$ can be represented by the end deflection, $\delta$ and the strip length, $l$.

$$
\delta=\frac{l^{2}}{2 R}
$$

In measurement of residual stress, the bending-beam method was employed. ${ }^{3}$ As the bending of specimen proceeded during drying, its amount was measured by detection of the position of reflected beam in detector, as depicted in Figure 1. The reflected beam position difference exhibited by position intensity difference $\Delta$ was proportional to the end deflection $\delta .^{3}$ The stress analyzer was supplemented by the interferometer system, since the solution of eq 2 required the value of film thickness as well as the radius of curvature.

The stress analyzer was composed of a heating system that could raise temperature up to $450^{\circ} \mathrm{C}$, a $8 \mathrm{~mW}$ $\mathrm{He}-\mathrm{Ne}$ Laser I (model 1134P, Uniphase), a position sensitive detector (model 1239, UDT sensors), and an oscilloscope (model OS3040, LG Precision). The heating system was composed of a heating coil and a thermostat (model 3000, LFE Instruments) with $\mathrm{N}_{2}$ gas purge line. Each stress measurement was performed at 30,60 , and $80^{\circ} \mathrm{C}$, respectively, inside a dark box on the optical table (Edmund Scientific Co.) to minimize external disturbance.

\section{Measurement of Young's Modulus}

Young's moduli of PI films were measured at room temperature using a universal tensile machine (UTS 10, UTS test system, Germany). Two types of PI samples, dry and NMP absorbed (solvent volume fraction $=0.32$ ) were used for their measurements. Curing and solvent absorption methods were the same as those mentioned above. Measurements were performed three times per each sample, and the average value was obtained for its determination.

\section{RESULTS AND DISCUSSION}

\section{Concentration Profiles and Film Thickness}

The equilibrium solvent volume fraction of PI film was 0.32 when it was immersed in NMP at $60^{\circ} \mathrm{C}$, and its thickness was $7.5 \mu \mathrm{m}$. Figure 2 illustrates evolution of film thickness during drying at various temperatures. Higher drying temperature enhanced the decreasing rate of film thickness due to the increasing solvent diffusion and evaporation rates.

If we set $x$-axis as the direction of thickness from substrate to film surface and assume that the film thickness is much smaller than its width and length, the diffusion and evaporation can be presumed to occur only in $x$-direction. Employing Fick's diffusion model ${ }^{11}$ under such assumptions, one can represent the relation regarding the solvent concentration $C_{1}$ as

$$
\frac{\partial C_{1}}{\partial t}=\frac{\partial}{\partial x}\left(D \frac{\partial C_{1}}{\partial x}\right)-\frac{\mathrm{d} C_{1}}{\mathrm{~d} x} u
$$

Here $D$ is the diffusion coefficient of the poly- 


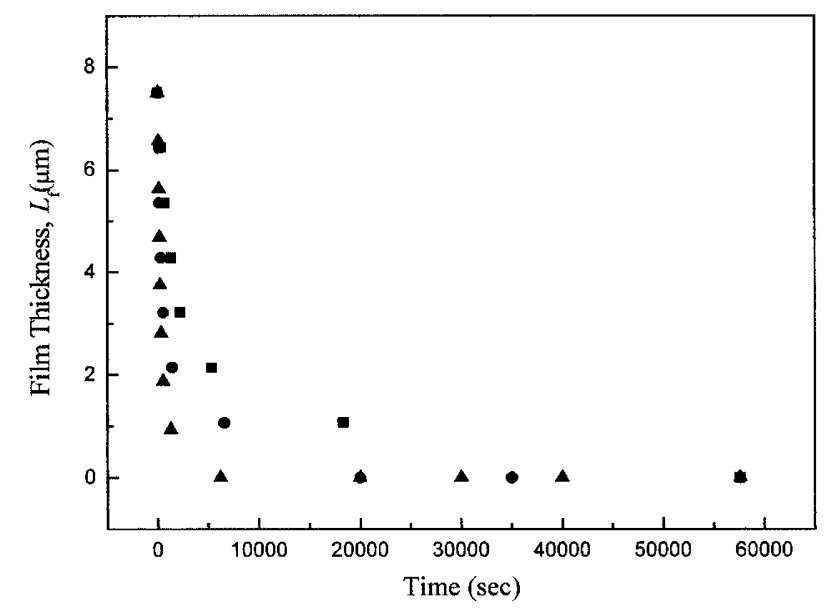

Figure 2. Thickness change of PI films during drying at varying temperatures of $30(\boldsymbol{\square}), 60(\boldsymbol{O})$, and $80^{\circ} \mathrm{C}(\boldsymbol{\Delta})$, respectively.

mer/solvent system and $u$ is bulk velocity. The hole free volume theory ${ }^{12-15}$ by Vrentas and Duda suggested the temperature and concentration dependence of $D$. As its relationship in this PI/NMP system had already been reported in the literature, ${ }^{4,5}$ we simply adopted it to the present analysis.

For the existence of unique solution of eq 4 , an initial and two boundary conditions should be supplemented. Initially, the solvent concentration inside film was uniform, hence

$$
C_{1}(x, 0)=C_{0}
$$

In addition, the boundary conditions could be written as eqs 6 and 7. Equation 6 expresses no mass transfer through film/substrate interface, and eq 7 implies the rate of solvent mass transfer from the film surface is equal to the rate of total solvent mass change $\frac{1}{A} \frac{\mathrm{d} M_{1 \mathrm{t}}}{\mathrm{d} t}$ in the film.

$$
\begin{aligned}
& \frac{\partial C_{1}}{\partial x}(0, t)=0 \\
& D \frac{\partial C_{1}}{\partial x}+C_{1} \frac{\partial L_{\mathrm{t}}}{\partial t}=\frac{1}{A} \frac{\mathrm{d} M_{1 \mathrm{t}}}{\mathrm{d} t} \quad \text { at } \quad x=L_{\mathrm{t}}
\end{aligned}
$$

The solution of eqs 4-7 resulted in solvent concentration profiles along the film thickness. The right hand side of eq 7 could be written in terms of a functional approximation of experimental film thickness data shown in Figure 2. The solution of eq 4 with the initial and boundary conditions given by eqs 5,6 , and 7 was obtained by the Crank-Nicholson's finite difference method. ${ }^{16}$ In this numerical scheme, the thickness was discretized into 100 sub-domains with a time step of $10^{-6} \mathrm{~s}$.

Figure 3 represents the calculated concentration profile during drying. It shows that the film surface moves toward the substrate and the concentration gradient decreases with drying time.

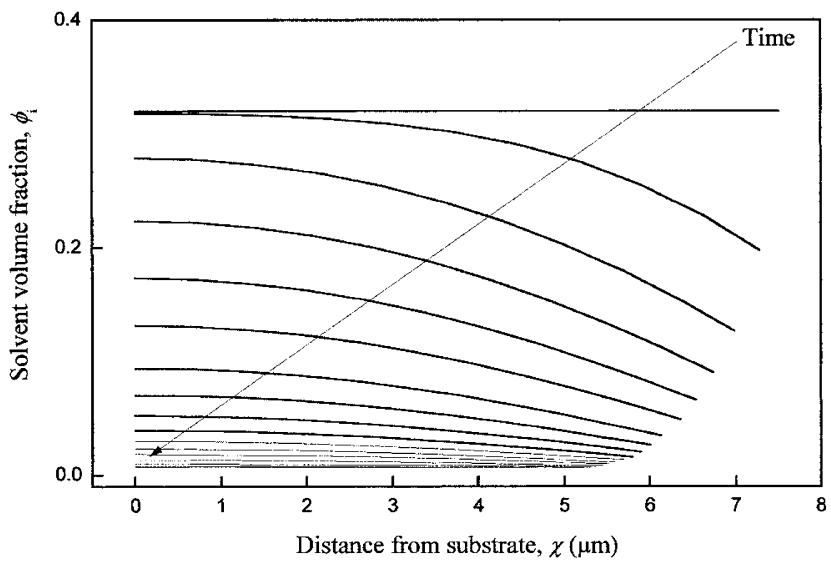

Figure 3. Solvent concentration profiles inside PI films during drying of polyimide films.

\section{Temperature Profiles}

When the polymer film coated on substrate was initially located in a heating block, the temperature difference between film and environment induced temperature gradient inside the film. Since the diffusion coefficient was dependent upon temperature, heat transport analysis was essential in order to estimate the mass transfer phenomena properly. In this system heat transfer occurred through two free surfaces of the film and substrate, as these surface temperatures in contact with thermocouples were isothermally controlled by external heat. Different temperature profiles were expected in film and substrate, once external heat was applied. For simple analysis of this heat transfer, we employed Fourier's equations ${ }^{11,17}$ as

$$
\begin{aligned}
& \rho_{\mathrm{f}} C_{\mathrm{pf}} \frac{\partial T_{\mathrm{f}}}{\partial t}=k_{\mathrm{f}} \frac{\partial^{2} T_{\mathrm{f}}}{\partial x^{2}} \\
& \rho_{\text {sub }} C_{\mathrm{psub}} \frac{\partial T_{\text {sub }}}{\partial t}=k_{\text {sub }} \frac{\partial^{2} T_{\text {sub }}}{\partial x^{2}}
\end{aligned}
$$

Here, $T_{\mathrm{f}}$ and $T_{\text {sub }}$ are temperatures, $\rho_{\mathrm{f}}$ and $\rho_{\text {sub }}$ densities, $C_{\mathrm{pf}}$ and $C_{\mathrm{psub}}$ heat capacities at constant pressure, and $k_{\mathrm{f}}$ and $k_{\text {sub }}$ heat transfer coefficients of film and substrate, respectively. Film properties were determined as the weight or volume additive values of two components, polymer and solvent.

To above eqs 8 and 9 , following conditions were given, that initial temperatures were equal to room temperature for both film and substrate:

$$
\begin{aligned}
& T_{\mathrm{f}}(x, 0)=T_{\mathrm{i}} \\
& T_{\text {sub }}(x, 0)=T_{\mathrm{i}}
\end{aligned}
$$

In addition, boundary conditions could be written as

$$
\begin{array}{ll}
T_{\mathrm{f}}=T_{\mathrm{b}} & \text { at } x=L_{\mathrm{t}} \\
T_{\text {sub }}=T_{\mathrm{b}} & \text { at } x=-L_{\text {sub }}
\end{array}
$$




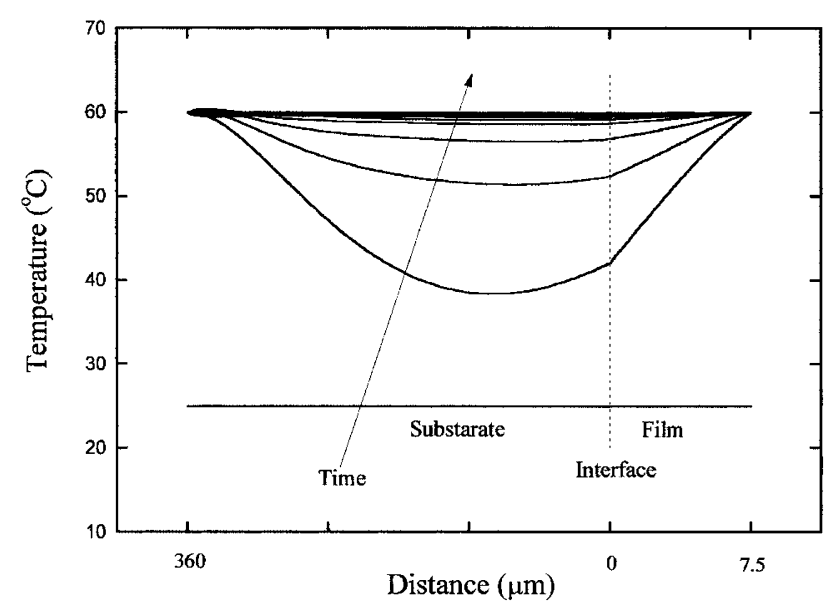

Figure 4. Temperature profiles inside substrate and film during drying of polyimide films.

$$
\begin{array}{ll}
T_{\mathrm{f}}=T_{\mathrm{sub}} & \text { at } x=0 \\
k_{\mathrm{f}} \frac{\partial T_{\mathrm{f}}}{\partial x}=k_{\mathrm{sub}} \frac{\partial T_{\mathrm{sub}}}{\partial x} & \text { at } x=0
\end{array}
$$

Here, $T_{\mathrm{b}}$ is the drying temperature. Equations 12 and 13 mean that the surface temperatures of film and substrate are equal to $T_{\mathrm{b}}$, eq 14 that film and substrate temperatures are the same at the film/substrate interface, and eq 15 that the heat transfer rate in substrate is equal to that in film at the interface.

Figure 4 illustrates the computation results on temperature distribution inside the film and substrate at the drying temperature of $60^{\circ} \mathrm{C}$. It was noted from Figure 4 that it took only about $1 \mathrm{~s}$ to reach equilibrium temperature. Since the completion time of drying process was around $20000 \mathrm{~s}$ at $60^{\circ} \mathrm{C}$, it was concluded that the local temperature difference in the film at the initial stage of isothermal drying operation was too small to affect the mass transfer. Similar results were obtained in the case of drying at 30 and $80^{\circ} \mathrm{C}$.

\section{Residual Stress}

Variation of solvent content inside the film leads to volume change. Due to the isotropy in normal strains, the volume change can be computed as follows:

$$
\frac{\mathrm{d} V}{V}=\frac{\mathrm{d} x}{x}+\frac{\mathrm{d} y}{y}+\frac{\mathrm{d} z}{z}=3 \varepsilon_{\mathrm{xx}}
$$

Equations 17 and 18 express the relationships among volume $V_{\mathrm{i}}$, concentration $C_{\mathrm{i}}$, and volume fraction $\phi_{\mathrm{i}}$ of each component. One can represent the volume change of film with respect to the fractional volume change of solvent as shown in eq 19.

$$
\begin{aligned}
& C_{1}=\rho_{1} \phi_{1}=\frac{m_{1}}{V}=\frac{m_{1}}{V_{1}+V_{2}}=\frac{\rho_{1} V_{1}}{V_{1}+V_{2}} \\
& \rho_{1} V_{1}=C_{1} V_{1}+C_{2} V_{2} \text { or } V_{1}=\frac{C_{1} V_{2}}{\rho_{1}-C_{1}}
\end{aligned}
$$

$$
\begin{aligned}
\frac{\mathrm{d} V}{V} & =\frac{\mathrm{d} V_{1}}{V_{1}+V_{2}}=\mathrm{d} \ln \left(V_{1}+V_{2}\right) \\
& =\mathrm{d} \ln \left(\frac{C_{1} V_{2}+\rho_{1} V_{2}-C_{1} V_{2}}{\rho_{1}-C_{1}}\right) \\
& =\mathrm{d} \ln \left(\frac{\rho_{1} V_{2}}{\rho_{1}-\rho_{1} \phi_{1}}\right)=\mathrm{d} \ln \left(\frac{V_{2}}{1-\phi_{1}}\right) \\
& =\mathrm{d} \ln V_{2}-\mathrm{d} \ln \left(1-\phi_{1}\right) \\
& =\frac{\mathrm{d} \phi_{1}}{1-\phi_{1}} \approx \frac{\Delta \phi_{1}}{1-\phi_{1}}
\end{aligned}
$$

The comparison of eq 16 with eq 19 yields the strain induced by solvent concentration change in the film during drying:

$$
\varepsilon_{\mathrm{xx}}=\varepsilon_{\mathrm{dry}}=\frac{\Delta \phi_{1}}{3\left(1-\phi_{1}\right)}=\frac{\phi_{1}-\phi_{1}^{\mathrm{i}}}{3\left(1-\phi_{1}\right)}
$$

Here, $\phi_{1}^{\mathrm{i}}$ is the initial solvent volume fraction in film. The total strain of film also contains the strain resulting from bending of the film. The film coated on substrate is strained by bending force $F_{\mathrm{f}}$, associated with the bending moment during drying process. The strain induced by this bending force $\varepsilon_{\text {bend }}$ is given by eq 21 , and thus the total strain $\varepsilon_{\mathrm{f}}$ by eq 22 .

$$
\begin{aligned}
& \varepsilon_{\text {bend }}=\frac{F_{\mathrm{f}}\left(1-\gamma_{\mathrm{f}}\right)}{E_{\mathrm{f}} L_{\mathrm{f}} w} \\
& \varepsilon_{\mathrm{f}}=\varepsilon_{\text {dry }}+\varepsilon_{\text {bend }} \\
& =\frac{\phi_{1}-\phi_{1}^{\mathrm{i}}}{3\left(1-\phi_{1}\right)}+\frac{F_{\mathrm{f}}\left(1-\gamma_{\mathrm{f}}\right)}{E_{\mathrm{f}} L_{\mathrm{f}} w}
\end{aligned}
$$

The bending force $F_{\mathrm{f}}$ also generates the strain of substrate as follows:

$$
\varepsilon_{\mathrm{sub}}=-\frac{F_{\mathrm{f}}\left(1-\gamma_{\mathrm{sub}}\right)}{E_{\mathrm{sub}} L_{\mathrm{sub}} w}
$$

Since the film is coated onto the substrate surface, both strains should be equal, that is, $\varepsilon_{\mathrm{f}}=\varepsilon_{\mathrm{sub}}$, and this fact yields for the bending force as follows:

$$
F_{\mathrm{f}}=\frac{\frac{-\left(\phi_{1}-\phi_{1}^{\mathrm{i}}\right)}{3\left(1-\phi_{1}\right)}}{\frac{1-\gamma_{\mathrm{f}}}{E_{\mathrm{f}} L_{\mathrm{f}} w}+\frac{1-\gamma_{\mathrm{sub}}}{E_{\mathrm{sub}} L_{\mathrm{sub}} w}}
$$

In above equation, since the thickness and modulus of substrate are much higher than those of film, i.e., $L_{\text {sub }} E_{\text {sub }} /\left(1-/ \gamma_{\text {sub }}\right)>>L_{\mathrm{f}} E_{\mathrm{f}} /\left(1-\gamma_{\mathrm{f}}\right)$, we can introduce the residual stress by the following approximation:

$$
\begin{aligned}
\sigma\left(\phi_{1}\right) & =\frac{F_{\mathrm{f}}}{L_{\mathrm{f}} w} \approx \frac{-\left(\phi_{1}-\phi_{1}^{\mathrm{i}}\right)}{3\left(1-\phi_{1}\right)} \times \frac{E_{\mathrm{f}}}{1-\gamma_{\mathrm{f}}} \\
& =\frac{-\Delta \phi_{1} E_{\mathrm{f}}}{3\left(1-\phi_{1}\right)\left(1-\gamma_{\mathrm{f}}\right)}
\end{aligned}
$$




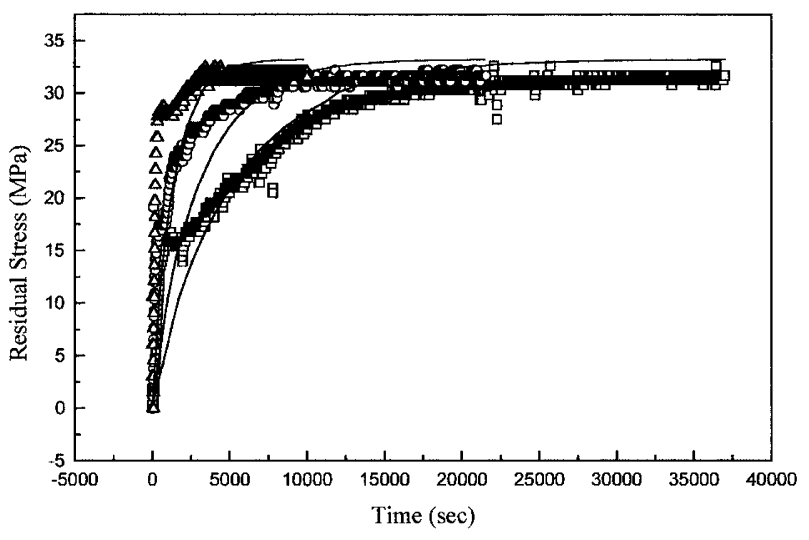

Figure 5. Residual stress of polyimide films during drying at varying temperatures of $30(\square), 60(\bigcirc)$, and $80^{\circ} \mathrm{C}(\triangle)$ from bottom to top; symbols (experimental data) and curves (theoretical results).

In order to compute the stress using eq 25, the solvent volume fraction was required. As the local solvent concentration varies along the thickness as shown in Figure 3, the stress is also position dependent. In order to simplify the calculation and compare the estimation with experimental data properly, we used average solvent volume fraction, $\overline{\phi_{1}}$ instead of local solvent volume fraction $\phi_{1}$ at each time. $\overline{\phi_{1}}$ was calculated by integration of $\overline{\phi_{1}}$ given by Figure 3 through the film thickness.

$$
\overline{\phi_{1}} \equiv\left\langle\phi_{1}\right\rangle=\frac{1}{L_{\mathrm{f}}} \int_{0}^{L_{\mathrm{f}}} \phi_{1} \mathrm{dz} \quad \text { at time } t .
$$

The resultant behavior of (average) residual stress is shown in Figure 5, where the bending curvature $R$ and film thickness $L_{\mathrm{f}}$ have been simultaneously measured in experiments. As the drying temperature increased, the duration time to reach equilibrium stress decreased, and it almost coincided with the duration time to equilibrium thickness shown in Figure 2. The equilibrium stress did not show much difference for each specimen. In Figure 5, the lines indicate the stress calculated theoretically from eq 25 with the modulus of film $E_{\mathrm{f}}$ of $2.0 \times 10^{8} \mathrm{~Pa}$ determined by best fit of theoretical calculation to experimental data.

The Young's moduli of dry and NMP absorbed PI films experimentally measured were $3.7 \times 10^{9} \mathrm{~Pa}$ and $6.9 \times 10^{8} \mathrm{~Pa}$ respectively. It means that the platicization effect by incorporation of about $32 \mathrm{vol} \%$ of NMP induced the reduction of room temperature modulus of PI more than 5 times. In literature, the typical modulus of PMDA-ODA type PI film was reported as $2.0 \times 10^{9} \mathrm{~Pa}$ at room temperature. Thus, the possible reasons that the modulus predicted from the present stress measurement was a little lower than the experimental or literature value were i) the solvent incorporated in films acted as plasticizer, ii) the drying temperatures at which the modulus was determined was higher than room temper- ature, and iii) the substitution of average concentration for $\phi_{1}$ in eq 25 might produce some erroneous calculation of stress.

\section{CONCLUSION}

The residual stress in polyimide films was measured with solvent evaporation and thickness change during drying, and their relationships were explained by heat and mass transfer and bending moment theories. We also verified the validity of the theory suggested in this work by the comparison of the residual stress theoretically computed with experimental data, and accordingly we were able to predict average modulus of the PI film. During drying process, the rate of heat transfer to the film and substrate was high enough to neglect the temperature dependence of the residual stress as well as diffusion coefficient. Higher drying temperature reduces time required for the film to reach equilibrium, and the instant at which equilibrium residual stress is attained, almost coincides with the moment of cessation of thickness variation.

Acknowledgment. This work was supported by the Korea Science and Engineering Foundation Grant 9811109-050-2.

\section{LIST OF SYMBOLS}

Symbols :

$C_{\mathrm{pf}}=$ specific heat of film at constant pressure $\left[\mathrm{J} \mathrm{g}^{-1}\right.$ $\mathrm{K}^{-1}$ ]

$C_{\mathrm{psub}}=$ specific heat of substrate at constant pressure $[\mathrm{J}$ $\mathrm{g}^{-1} \mathrm{~K}^{-1}$ ]

$C_{1}=$ solvent concentration inside the film $\left[\mathrm{g} \mathrm{cm}^{-3}\right]$

$C_{0}=$ initial solvent concentration inside the film $[\mathrm{g}$ $\mathrm{cm}^{-3}$ ]

$D=$ mutual diffusion coefficient $\left[\mathrm{cm}^{2} \mathrm{~s}^{-1}\right]$

$E_{\mathrm{f}} \quad=$ Young's modulus of film [MPa]

$E_{\text {sub }}=$ Young's modulus of substrate [MPa]

$F_{\mathrm{f}} \quad=$ force acting on film $[\mathrm{N}]$

$F_{\text {sub }}=$ force acting on substrate $[\mathrm{N}]$

$k_{\mathrm{f}}=$ thermal conductivity of film $\left[\mathrm{W} \mathrm{m}^{-1} \mathrm{~K}^{-1}\right.$ ]

$k_{\mathrm{sub}}=$ thermal conductivity of substrate $\left[\mathrm{W} \mathrm{m}^{-1} \mathrm{~K}^{-1}\right.$ ]

$l=$ length of film/substrate composite strip [ $\mu \mathrm{m}]$

$L_{0} \quad=$ initial film thickness [ $\mu \mathrm{m}$ ]

$L_{1 \mathrm{t}}=$ film thickness attributed to the presence of solvent at arbitrary drying time, $t[\mu \mathrm{m}]$

$L_{\text {sub }}=$ substrate thickness [ $\left.\mu \mathrm{m}\right]$

$L_{\mathrm{t}} \quad=$ film thickness at arbitrary drying time, $t[\mu \mathrm{m}]$

$n_{\mathrm{s}} \quad=$ refractive index of the solvent

$R \quad$ = bending curvature $[\mu \mathrm{m}]$

$T \quad=$ temperature $[\mathrm{K}]$

$T_{1} \quad=$ convenient reference temperature $[\mathrm{K}]$ 
$T_{\mathrm{b}} \quad=$ temperature in bulk atmosphere [K]

$T_{\mathrm{f}} \quad=$ temperature inside polymer film [K]

$T_{\mathrm{i}} \quad=$ initial temperature of polymer system $[\mathrm{K}]$

$T_{\text {sub }}=$ temperature inside substrate $[\mathrm{K}]$

$t \quad=$ time [s]

$V_{1}=$ solvent volume $\left[\mathrm{cm}^{3}\right]$

$V_{2} \quad=$ polymer volume $\left[\mathrm{cm}^{3}\right]$

$w=$ width of film/substrate composite [ $\mu \mathrm{m}]$

$x=$ distance from the substrate/film interface to film surface $[\mu \mathrm{m}]$

$\delta \quad=$ end deflection $[\mu \mathrm{m}]$

$\phi_{1} \quad=$ volume fraction of solvent

$\bar{\phi}_{1}=$ average volume fraction of solvent

$\phi_{1}^{\mathrm{i}}=$ initial volume fraction of solvent

$\phi_{2}=$ volume fraction of polymer

$\gamma_{\mathrm{f}}=$ Poisson's ratio of film

$\gamma_{\text {sub }}=$ Poisson's ratio of substrate

$\lambda_{\text {beam }}=$ wavelength of laser $[\mu \mathrm{m}]$

$\rho_{\mathrm{f}} \quad=$ density of film $\left[\mathrm{g} \mathrm{cm}^{-3}\right]$

$\rho_{\text {sub }}=$ density of substrate $\left[\mathrm{g} \mathrm{cm}^{-3}\right]$

\section{REFERENCES}

1. C. C. Chao and K. D. Scholz, "Proc. 38th Electronic Component Conference", 1998, p 276.

2. R. A. Larsen, IBM J Res Dev, 26, 268 (1980).

3. H. J. Kook and D. Kim, J. Mater. Sci., 35, 2949 (2000).

4. K. S. Park and D. Kim, Polymer (Korea), 25, 194 (2000).
5. K. S. Park and D. Kim, Polym. J., 32, 415 (2000).

6. K. Sato, K. Mukai, S. Harada, A. Saeki, T. Kimura, T. Okubo, I. Ishi, and I. Shimizu, "IEEE Trans on Hybrid and Packaging”, 1973, vol. PHP-9, pp 173.

7. J. C. Coburn and M. T. Pottiger, "In Polyimides: Fundamentals and Applications", M. K. Ghosh and K. L. Mittal, Ed., Marcel Dekker, Inc., New York, N.Y., 1996, pp 207-247.

8. H. Satou and D. Makino, "Polyimides for Electric Applications”, Hitachi Chemical Co., Ltd., Ibaraki, Japan, 1993.

9. K. L. Saenger and H-M. Tong, in "In New Characterization Techniques for Thin Polymer Films" H-M. Tong and L. T. Nguyen, Ed., John Wiley \& Sons, Inc., New York, N.Y., 1990, p 95.

10. M. Ohring, "The Materials Science of Thin Films", Academic Press, Inc., New York, N.Y., 1992, pp 405-438.

11. R. B. Bird, W. E. Stewart, and E. N. Lightfoot, "Transport Phenomena", Part III, John Wiley \& Sons, Inc., New York, N.Y., 1960.

12. J. L. Duda, J. S. Vrentas, S. T. Ju, and H. T. Liu, AIChE J, 28, 279 (1982).

13. J. M. Zielinski and J. L. Duda, AIChE J., 38, 405 (1992).

14. J. S. Vrentas and C. M. Vrentas, J. Polym. Sci., Polym. Phys. Ed., 32, 187 (1994).

15. J. S. Vrentas, J. L. Duda, H. C. Ling, and A. C. Hou, J. Polym. Sci., Polym. Phys. Ed., 23, 289 (1985).

16. W. H. Press, B. P. Flannery, S. A. Teukolsky, and W. T. Vetterling, "Numerical Recipes in C", Cambridge University Press, Cambridge, 1988, pp 636-688.

17. W. L. McCabe, J. C. Smith, and P. Harriott, "Unit Operation of Chemical Engineering”, 5th, ed, McGraw-Hill, New York, N.Y., 1993, pp 285-492. 